\title{
Pectus up: la nueva técnica de tracción para pectus excavatum. Primera cirugía en el noreste de México
}

\author{
Pectus up: the new traction technique for pectus excavatum. First surgery in Northeast of \\ Mexico
}

\section{Edson R. Marcos-Ramírez*, Jorge A. Garza-Cerna, Ignacio Fonseca-Sada, Alejandra Téllez-Aguilera,} Samuel Salazar-Palafox, Fernando Montes-Tapia, Manuel Wong-Jaen y Gerardo E. Muñoz-Maldonado Servicio de Cirugía General, Hospital Universitario Dr. José Eleuterio González, Universidad Autónoma de Nuevo León, Monterrey, Nuevo León, México

\section{Resumen}

El pectus excavatum es la deformidad de la caja torácica frecuente, siendo el sexo masculino el mayormente afectado. Presentamos el caso de un varón de 16 años con pectus excavatum e índice de Haller de 4, a quien se somete a cirugía de pectus up con sistema de tracción esternal externa y fijación con tornillos. Se obtuvo una muy buena respuesta funcional y estética en su posoperatorio. La cirugía de pectus up como método poco invasivo da buenos resultados posoperatorios.

Palabras clave: Extratorácico. Técnica. Tracción. Pectus excavatum.

\begin{abstract}
The pectus excavatum is the most frequent deformity of the rib cage, with the male sex being the most affected. We present the case of a 16-year-old male with the presence of pectus excavatum with a Haller's index of 4 who undergoes pectus up surgery with an external sternal traction system and screw fixation. A very good functional and aesthetic response was obtained. The pectus up surgery as a less invasive technique has good postoperative results.
\end{abstract}

Key words: Extrathoracic. Technique. Traction. Pectus excavatum.

\section{Introducción}

El pectus excavatum (PE) es la malformación torácica congénita más frecuente, afectando a uno de cada 400 nacimientos, predominantemente en hombres, y se encuentra relacionado con el crecimiento del cartílago costo-condral. Puede ser simétrico (involucrando el esternón en el plano frontal con deformidad en el mismo lado en el plano sagital) o asimétrico ${ }^{1}$. Las etiologías del PE son múltiples: idiopática ( $50 \%$ de los casos), hereditaria ( $40 \%$ de los casos) $)^{2}$ o un padecimiento de la colágena como el síndrome de Marfan ( $5 \%$ de los casos) o la enfermedad de Ehler-Danlos (1\% de los casos) ${ }^{3}$. En los niños,

\section{Correspondencia:}

*Edson R. Marcos-Ramírez

Avda. Francisco I Madero, s/n 
la progresión de la deformidad torácica está relacionada con el crecimiento y se observa una tendencia a empeorar con la edad. El riesgo progresivo es potencialmente mayor al llegar a la pubertad debido a que la circunferencia torácica se incrementa un $50 \%$ durante esta etapa.

Actualmente, la evaluación paraclínica de la gravedad del PE se basa en el índice de Haller por tomografía computada (TC). Este índice proporciona la relación del diámetro transversal del tórax respecto a su diámetro anteroposterior en el punto donde la deformidad es más pronunciada ${ }^{4}$.

Existen diversos procedimientos quirúrgicos para tratar este padecimiento, tales como los descritos por Ravitch ${ }^{5}$ y Bedouelle ${ }^{6}$ en 1955 y 1964, respectivamente, en los cuales se realizan varias osteotomías en el esternón y los arcos costales, y se colocan una o varias barras metálicas en el tórax para mantener la reconstrucción. Estas técnicas tienen la desventaja de la presencia de cicatrices sustanciales, lo que dificulta su aplicación en el plano cosmético, además de ser particularmente invasivas para los pacientes, y comporta los riesgos que implica el abordar de esta manera la caja torácica, como son las lesiones de órganos intratorácicos.

La técnica descrita por Nuss es un abordaje de mínima invasión en el cual se eleva el esternón colocando una barra metálica restroesternal guiándose por toracoscopia. Esta técnica se basa en la flexibilidad del tórax de los pacientes jóvenes y presenta la ventaja de corregir la deformidad anatómica torácica con un abordaje menos invasivo y con un seguimiento posoperatorio sencillo ${ }^{7}$. Actualmente es considerado el procedimiento de elección en todo el mundo para el tratamiento del PE, y numerosos reportes han demostrado los excelentes resultados a largo plazo y su seguridad $^{8}$.

La incidencia de complicaciones mayores o menores con este procedimiento se han reportado en bajos rangos $(2-27 \%)^{7,9-11}$. De acuerdo con los datos de la literatura, la función pulmonar no se mejora objetivamente ${ }^{12,13}$, además de que la extracción del implante puede ser difícil con este tipo de cirugía.

Inspirado en los mismos principios del procedimiento de Nuss, el levantamiento esternal o pectus up puede ser posible a través de una tracción externa, sin necesidad de colocar una barra intratorácica; de esta manera se evita la invasión al mediastino o a la cavidad pleural. Para este propósito, se diseñó una placa para la implantación extratorácica con un sistema de tracción asociado. Esta placa tiene la forma y las medidas específicas para soportar y distribuir las cargas necesarias sobre el defecto. El sistema de tracción consiste en un tornillo y una tuerca que se aprietan progresivamente en la placa del PE hasta que se logra la reducción del defecto ${ }^{14}$.

La principal ventaja demostrada con este abordaje es evitar la invasión al mediastino o a la cavidad pleural para así reducir las principales complicaciones de la toracoplastia mínimamente invasiva de Nuss y el riesgo de lesiones en órganos vitales ${ }^{15,16}$.

Las indicaciones específicas para este tipo de reparación del PE son las siguientes: índice de Haller > 3.25 con compresión cardiaca o pulmonar, pruebas de función pulmonar que demuestren un deterioro obstructivo o restrictivo, evaluación cardiológica que demuestre compresión cardiaca, prolapso de la válvula mitral, soplos o anormalidades en la conducción, documentación de la progresión de la deformidad conforme avanza la edad en asociación con el desarrollo o empeoramiento de los síntomas (disnea, intolerancia al ejercicio, palpitaciones y dolor torácico) ${ }^{17}$.

Presentamos un caso de PE en el que se realizó la técnica de levantamiento esternal o pectus up, siendo la primera cirugía pediátrica de este tipo en el noreste de México.

\section{Caso clínico}

Varón de 16 años, producto de primera gestación, nacido vía cesárea sin complicaciones, 1.70 metros de estatura, $47 \mathrm{~kg}$ de peso, índice de masa corporal de 16, estudiante, con antecedente de numerosas hospitalizaciones por cuadros respiratorios durante la infancia, diagnóstico de PE y flaring costal visible desde los 10 años (Fig. 1).

Su sintomatología respiratoria consiste en disnea después de 5 minutos de actividad física leve. Se le realizan pruebas de función pulmonar que reportan restricción leve, la Capacidad Vital Forzada (FVC) de 80.9 con un Volumen de Espiración Forzado en 1 segundo/FVC (FEV1/FVC) ratio de 110.5. La ecografía transtorácica evidencia un corazón estructuralmente sano, con anillo aórtico de $17 \mathrm{~mm}$ y anillo pulmonar de $20 \mathrm{~mm}$, sin defectos de la segmentación cardiaca, retornos venosos sistémicos y pulmonares normales, con concordancia auriculoventricular y ventriculoauricular.

La TC de tórax reporta depresión posterior del apéndice xifoides y últimos cartílagos costales en relación con $\mathrm{PE}$, con diámetro anteroposterior de $52.2 \mathrm{~mm}$ y transverso de $212.33 \mathrm{~mm}$, resultando un índice de Haller de 4, que causa una ligera 


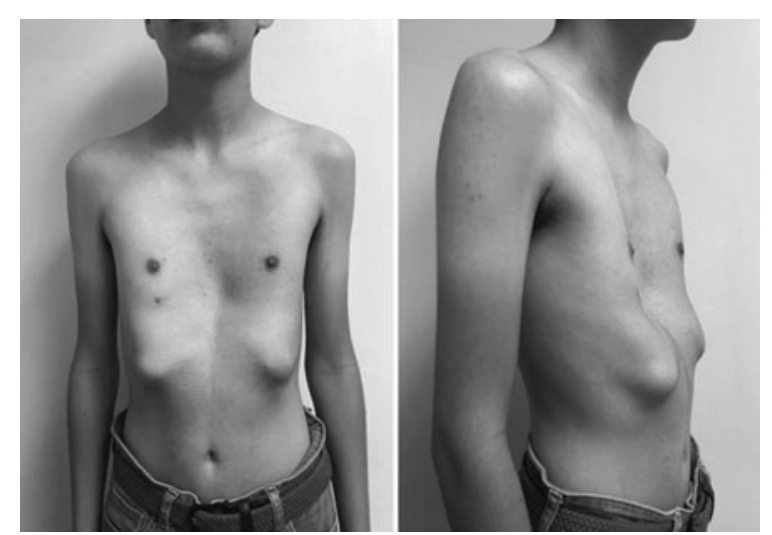

Figura 1. Pectus excavatum, fotografía prequirúrgica del paciente.

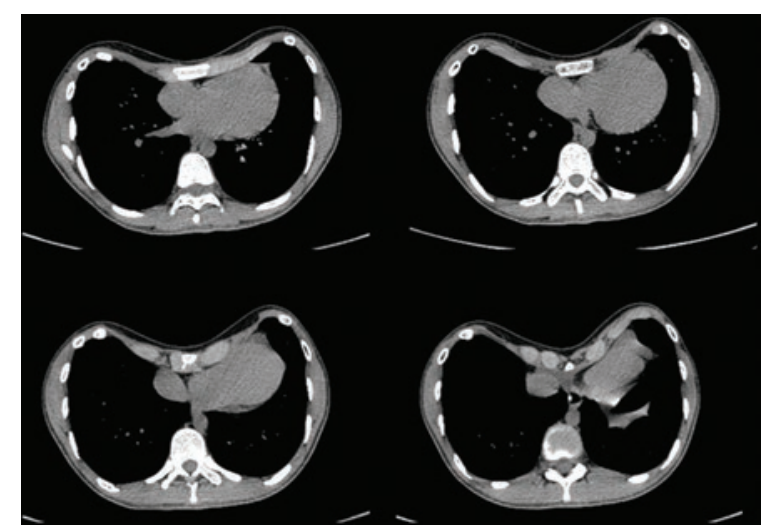

Figura 2. Tomografía axial computada de nuestro paciente, con un diámetro anteroposterior de $52.2 \mathrm{~mm}$ y un diámetro transverso de $212.33 \mathrm{~mm}$, y un índice de Haller de 4. Se observa una ligera compresión de las cavidades derechas y el desplazamiento del corazón a la izquierda.

compresión de las cavidades derechas y desplazamiento del corazón a la izquierda (Fig. 2).

Se decide realizar corrección quirúrgica del defecto mediante la técnica de pectus up, siendo necesaria una elevación esternal de $2 \mathrm{~cm}$. Se realizó el abordaje en el punto de mayor depresión del esternón, además de los dos puntos de abordaje subcostales para el sistema de tracción con el cual el defecto fue progresivamente reducido; después de este punto, se fija el esternón con tornillos y después se retira el sistema de tracción. Las ventanas son cerradas con sutura absorbible para mejorar la estética.

La cirugía tiene buenos resultados, sin complicaciones tempranas ni tardías y con una recuperación con dolor leve sin mayor necesidad de analgésicos potentes. En la figura 3 se muestran los resultados anatómicos de la cirugía de reconstrucción durante los primeros días posoperatorios.

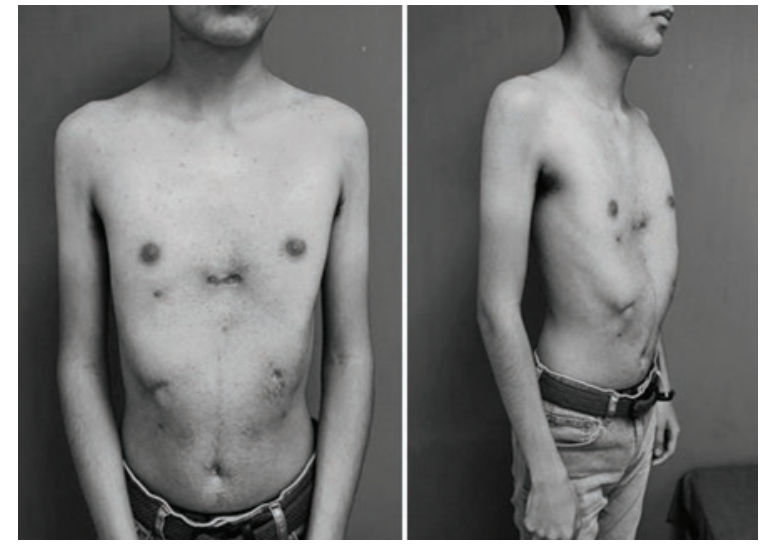

Figura 3. Resultado de la cirugía, 1 semana posterior al alta médica.

Actualmente el paciente lleva una vida normal y se encuentra asintomático con buenos resultados funcionales, sin la sintomatología respiratoria inicial y con un resultado estético favorable para el paciente a 18 meses de la cirugía.

\section{Discusión}

La cirugía pectus up es una técnica actual y pionera en la cirugía de reconstrucción del PE por mínima invasión. En Europa y Norteamérica se ha iniciado su práctica como primera línea terapéutica, mientras que en América Latina se empiezan a operar casos aislados reportados con muy buenos resultados. En México, la cirugía de pectus up comienza a realizarse, y por ello reportamos la primera llevada a cabo en el noreste del país. Siendo pacientes pediátricos los que habitualmente se presentan con PE, esta técnica de mínima invasión que reduce las posibles complicaciones importantes intratorácicas resulta un procedimiento ideal y que empieza a cobrar fuerza en países más desarrollados como primera línea de tratamiento para el PE. Tuvimos un buen resultado con este procedimiento, tanto funcional como estético, el paciente terminó satisfecho con los cambios de la cirugía y la sintomatología se resolvió en la primera semana posoperatoria. Debido a que es un procedimiento relativamente sencillo, y respaldados por lo reportado en la literatura ${ }^{14}$, la curva de aprendizaje es corta en comparación con otro tipo de técnicas de reparación, beneficiados con la baja frecuencia de las complicaciones que se observan con técnicas más invasivas ${ }^{16}$. El objetivo principal de este reporte es ofrecer otra opción terapéutica al arsenal quirúrgico para el tratamiento del PE. Durante la técnica hay que tener un 
especial cuidado en colocar la placa de retracción cerca del apéndice xifoides y realizar la tracción con un ángulo de $90^{\circ}$ para evitar fracturas del plano esternal o costal.

\section{Conclusión}

Con este caso reportado iniciamos en nuestro centro hospitalario el uso de la técnica de pectus up para el PE, esperando que sea el primero de los muchos pacientes que pueden beneficiarse de esta técnica en nuestro país. Los beneficios que conlleva esta novedosa técnica hacen de ella un procedimiento ideal para el manejo de pacientes pediátricos con $\mathrm{PE}$. Con el reporte del caso, nuestro objetivo como equipo de trabajo quirúrgico es aumentar la difusión y la experiencia en la literatura nacional para que en un futuro próximo tengamos estudios clínicos más elaborados que evalúen esta prometedora técnica de reducción extratorácica.

\section{Agradecimientos}

Los autores agradecen al equipo de trabajo y los pacientes del hospital-escuela, que son la razón de ser de nuestra labor.

\section{Financiamiento}

Los autores declaran que no hubo fuente de financiamiento para la realización de este trabajo.

\section{Conflicto de intereses}

Los autores declaran que no hubo ningún conflicto de intereses.

\section{Responsabilidades éticas}

Protección de personas y animales. Los autores declaran que los procedimientos seguidos se conformaron a las normas éticas del comité de experimentación humana responsable y de acuerdo con la Asociación Médica Mundial y la Declaración de Helsinki.

Confidencialidad de los datos. Los autores declaran que han seguido los protocolos de su centro de trabajo sobre la publicación de datos de pacientes.

Derecho a la privacidad y consentimiento informado. Los autores han obtenido el consentimiento informado de los pacientes y/o sujetos referidos en el artículo. Este documento obra en poder del autor de correspondencia.

\section{Bibliografía}

1. Felts E, Jouve JL, Blondel B, Launay F, Lacroix F, Bollini G. Child pectus excavatum: correction by minimally invasive surgery. Orthop Traumatol Surg Res. 2009;95:190-5.

2. Creswick HA, Stacey MW, Kelly RE, Gustin T, Nuss D, Harvey H, et al. Family study of the inheritance of pectus excavatum. J Pediatr Surg. 2006;41:1699-703.

3. Albejano MR, Del Campo IM, Jové MG. Pectus excavatum. FMC Form Medica Contin Aten Primaria. 2013;20:556-7.

4. Haller JA Jr, Kramer SS, Lietman SA. Use of CT scans in selection of patients for pectus excavatum surgery: a preliminary report. J Pediatr Surg. 1987;22:904-6.

5. Ravitch MM. The operative treatment of pectus excavatum. J S C Med Assoc. 1955;51:244-9.

6. Bedouelle J. Diaphragm lengthening technique. Complementary stage of plastic correction. Rev Chir Orthop. 1964;50:476-82.

7. Fonkalsrud EW, Beanes S, Hebra A, Adamson W, Tagge E. Comparison of minimally invasive and modified ravitch pectus excavatum repair. $J$ Pediatr Surg. 2002;37:413-7.

8. Nuss D. Minimally invasive surgical repair of pectus excavatum. Semin Pediatr Surg. 2008;17:209-17.

9. Hansen MK. Is the grass greener? ASDC J Dent Child. 1978;45:184.

10. Fallon SC, Slater BJ, Nuchtern JG, Cass DL, Kim ES, Lopez ME, et al. Complications related to the Nuss procedure: Minimizing risk with operative technique. J Pediatr Surg. 2013;48:1044-8.

11. Castellani C, Schalamon J, Saxena AK, Höellwarth ME. Early complications of the Nuss procedure for pectus excavatum: a prospective study. Pediatr Surg Int. 2008;24:659-66.

12. Borowitz D, Cerny F, Zallen G, Sharp J, Burke M, Gross K, et al. Pulmonary function and exercise response in patients with pectus excavatum after nuss repair. J Pediatr Surg. 2003;38:544-7.

13. Aronson DC, Bosgraaf RP, Merz EM, Van Steenwijk RP, Van Aalderen WMC, Van Baren R. Lung function after the minimal invasive pectus excavatum repair (Nuss procedure). World J Surg. 2007;31:1518-22.

14. Bardají C, Cassou L. Taulinoplasty: the traction technique - a new extrathoracic repair for pectus excavatum. Ann Cardiothorac Surg. 2016;5:519-22.

15. Helly RE, Mellins RB, Shamberger RC, Mitchell KK, Lawson ML, Oldham KT, et al. Multicenter study of pectus excavatum, final report: complications, static/exercise pulmonary function, and anatomic outcomes. J Am Coll Surg. 2013;217:1080-9.

16. Schaarschmidt K, Lempe M, Schlesinger F, Jaeschke U, Park W, Polleichtner S. Lessons learned from lethal cardiac injury by nuss repair of pectus excavatum in a 16-year-old. Ann Thorac Surg. 2013:95:1793-5.

17. Nuss D, Kelly RE. Indications and technique of nuss procedure for pectus excavatum. Thorac Surg Clin. 2010;20:583-97. 